\title{
On the impact of RLC layer configuration parameters in UMTS Internet access
}

\author{
J. M. Cano-García, E. González-Parada and E. Casilari-Pérez. \\ Departamento de Tecnología Electrónica. Universidad de Málaga. 29071. Málaga. Spain. Email: cano@dte.uma.es.
}

\begin{abstract}
The main goal of this paper is the analysis of the interaction between TCP and the UMTS link-level layer (RLC) to determine an optimal configuration for the parameters of both protocols. For this purpose, a web browsing simulation framework has been considered. This framework includes a web client accessing to remote HTTP servers through the UTRAN and Internet. The impact of the RLC configuration parameters on the behaviour of the multiple TCP connections simulated under this framework is analysed, showing their effect on web traffic performance.
\end{abstract}

\section{INTRODUCTION}

Providing efficient Mobile Internet Access is one of the main goals of third generation (3G) mobile systems. However, a pivotal issue in the performance of Internet access is the behaviour of the Transmission Control Protocol (TCP). TCP is used in most Internet applications to support end to end reliable data transfer, but it also provides a congestion control mechanism, which is an essential factor to assure the stability of Internet traffic. However, TCP congestion control does not perform well in error-prone environments like radio cellular systems, basically due to the fact that it is based on the assumption that packet losses are a congestion symptom. This fact leads to an unnecessary reduction of the TCP transmission rate when packet are lost due to corruption in the radio interface. This issue has been the focus of an important research effort in the recent years, and several solutions have been proposed to address it [1].

TCP disability to discriminate between corruption and congestion losses usually make necessary the utilization of local retransmission mechanisms to hide radio losses from TCP, transparently providing a reliable data transmission channel. In UMTS (Universal Mobile Telecommunication Systems), this is accomplished by the RLC (Radio Link Control) protocol sublayer. However, other factors can also degrade TCP performance in radio access network as proven in recent research studies [2], [3]. In particular, Link-level retransmission mechanisms can lead to bandwidth and latency fluctuations caused by the variability of the time necessary to recover from different packet losses. This may also affect TCP performance and degrade the end-to-end QoS. In general, TCP interaction with lower layer mechanisms cannot be neglected and should be considered when designing the radio access network [4], [5].

Different works have been devoted to the analysis of the interaction between TCP and UMTS Radio Access Network (UTRAN) protocols, yielding different solutions to minimize the effect of losses, and bandwidth and latency fluctuations on TCP. Although cross-layer interaction analysis is often used to derive the different proposals, they are usually focused on a given sublayer. Thus, several studies propose TCP parameters and mechanisms tuning to improve its performance over the radio link constraints [6], [7], whereas other researchers focus their attention in adjusting the configuration of the UTRAN protocols to reduce the negative impact of the radio interface on TCP. In this second group, different strategies can also be also differentiated: several studies center on radio resource management and medium access control mechanisms [8], [9], whereas others are aimed to the study of RLC layer configuration and its effect on TCP traffic performance [10], [11], [12].

The present work is focuses on the interaction between TCP and the RLC sublayer. The main goal is to complete the previously cited contributions, which commonly limit the study to the impact of RLC parameters in bulk TCP traffic. In the present paper the analysis is expanded to evaluate the effect of RLC configuration in the QoS perceived by web browsing clients, which generate a more complex traffic pattern including both bulk and short-lived transfers.

This paper is structured as follows: in Section II, a summary of UMTS RLC layer mechanisms is presented and an overview of the background research on this subject is provided. The evaluation scenario and the simulation models used in the present study are described in section III. Section IV deals with the results of the evaluation of different RLC schemes. Finally, conclusions are drawn on section V.

\section{RLC AND RELATED RESEARCH OVERVIEW}

UMTS RLC sublayer [13] comprises all the mechanisms needed to the fragmentation and reassembly of upper layer data packets (Service Data Units, SDUs) into smaller data blocks (RLC Packet Data Units, PDUs) so that they can be transmitted through the radio interface by the lower layers. RLC can operate in three different modes: Transparent, Unacknowledged (UM) and Acknowledged Mode (AM). When configured in AM mode, the RLC implements an ARQ mechanism to alleviate PDU losses due to radio link transmission errors. For this purpose, the RLC specification defines a selective repeat sliding window procedure. According to this procedure the receiving RLC entity, under certain conditions, sends back status reports to the transmitting RLC entity of the other side, indicating the which PDUs have been correctly received and which ones are missing. Basing on this 


\begin{tabular}{|c|l|}
\hline \multicolumn{2}{|c|}{ Transmitter parameters } \\
\hline \hline Timer Poll Periodic & Poll request can be triggered periodically basing on this timer \\
\hline Poll PDU & Poll can be triggered every time a given number of PDUs are transmitted \\
\hline Poll Window & Poll is triggered when the number of unacknowledged PDUs outreaches a given fraction of the maximum transmission window \\
\hline Timer Poll & Timer to trigger the retransmission of a poll request if no status report is received in a given interval after sending a previous one \\
\hline Timer Poll Prohibit & Defines an inhibition interval after sending a poll request to avoid excessive polling \\
\hline Timer SDU Discard & Timer after which a SDU is discarded if it has not been successfully transmitted \\
\hline SDU Discard & SDU can also be discarded if the retransmissions on its PDUs have been foiled a given number of times \\
\hline Timer MRW Retransmit & Timer to trigger a new SDU discard request if no confirmation of a previous request has been received after a given interval \\
\hline \hline \multicolumn{2}{|c|}{ Receiver parameters } \\
\hline \hline Timer Status Periodic & Periodic timer to trigger status reports \\
\hline Timer Status Prohibit & Defines an inhibition interval after sending a status report \\
\hline Missing PDU Threshold & An status report is sent if the number of missing PDU exceeds a given limit \\
\hline
\end{tabular}

TABLE I

SUMMARY OF RLC LAYER PARAMETERS

information, the transmitting RLC entity decides either the retransmission of previously sent PDUs or the transmission of new ones. In order to avoid stall, the transmitting entity can also request a status report to the peer receiving entity by means of a poll indication bit in the AM PDUs. In that case, the receiver should responds immediately by sending back a status PDU to the transmitter. The UMTS specification defines several mechanisms to trigger both the polling mechanisms in the transmitter and status reports in the receiver, which yields a wide set of configuration parameters. A summary of these mechanism is provided in table $\mathrm{I}$, and a detailed description can be found in [13]. Apart form the parameters shown in table I, two other mechanisms exists to avoid deadlocks: The transmission of a polling request with the last PDU in the new transmission buffer and with the last PDU in the retransmission buffer

In addition to the parameters related to polling and status report triggering mechanisms, there are several parameters that governs SDU discarding (also presented in table I). The RLC specification determines that SDUs can be discarded in AM mode if they are not successfully transmitted after a certain time or after attempting a given number of retransmission. In order to do this, the RLC transmitter must send a Move Receiver Window (MRW) request to the receiver. The MRW request should be confirmed to complete the SDU discard and continue the normal transmission of the following SDUs.

As previously mentioned, several contributions on the impact of the RLC settings in TCP traffic can be found in the literature, but usually these studies are limited to one or two parameters and are executed in scenarios where only one or few TCP connections are simulated. In particular, [14] studies the influence of several link layer parameters, such as the RLC maximum number of retransmissions (i.e. SDU discard), radio link latency, and error pattern, over a single long-lived TCP connection depending on TCP window size. This study concludes that the maximum number retransmissions should be set to the higher available limit, since TCP tackles variable delay better than losses. Also, it is derived that the TCP window should be configured as high as possible but always bellow the network bandwidth-delay product to optimize RLC buffer occupancy. Similarly, [10] considers a single TCP connection, evaluating which factors affect buffer occupancy, deriving that the occupancy rises as the error rate in the radio interface goes higher or the Internet segment latency decreases. Besides, they conclude that as the RLC buffer size increases so does the TCP achieved goodput. In [12] and [15] analytical models both for the ARQ scheme and TCP are developed to evaluate the interaction between their mechanisms as a function of the variable channel conditions with different QoS constraints, focusing in particular on the configuration of the TCP MSS and the maximum number of ARQ retransmissions. As a result they propose an adaptative algorithm to fit the TCP and ARQ parameter configuration to the radio link circumstances. Similarly, [11] proposes an adaptative algorithm to adjust the number of maximum retransmission in the RLC layer, but using a simulation study to derive the results. However they finally conclude that although the adaptative tuning of the number of retransmission limit improves TCP performance, the gain is not significative, and therefore the better option is to adopt a fully reliable ARQ link layer.

The work presented here is intended to complete the previous contributions. In the present study, several RLC configuration parameters are evaluated in a simulation framework which considers a more realistic web traffic pattern consisting of a high number of TCP connections with different characteristics.

\section{A. Simulation Scenario}

The evaluation scenario has been chosen so that it is as realistic and representative as possible but intending to avoid an excessive simulation cost. Therefore, in order to evaluate RLC configuration impact on UMTS Internet access, an scenario involving web browsing sessions over a dedicated channel has been considered. It should be stated that RLC parameter configuration effect over the QoS might be different for other services with other features than web traffic. However, the simulation of web traffic has been selected rather than peer to peer o bulk transfers, because it is more suitable for Mobile Internet environments, where billing is based on data volume.

The architecture of the simulation model for the proposed scenario is depicted in fig. 1. Detailed computational simulation models of both the TCP and the RLC layer, comprising all their mechanisms, have been developed to perform a study of their interaction. Also, a realistic traffic model for WWW browsing has been included into the model to reproduce the characteristics of this traffic. On the other hand, the behaviour of other layers such as IP, Packet Adaptation (PDCP) and 
UMTS Core Network transport have been simplified. Packet transit through the Internet and the Core Network have been modelled as an additional fixed latency. Finally, MAC and physical layer are characterized by a transmission interval (TTI), the number of transport blocks delivered per TTI, the transport block size and the Bit Error Rate.

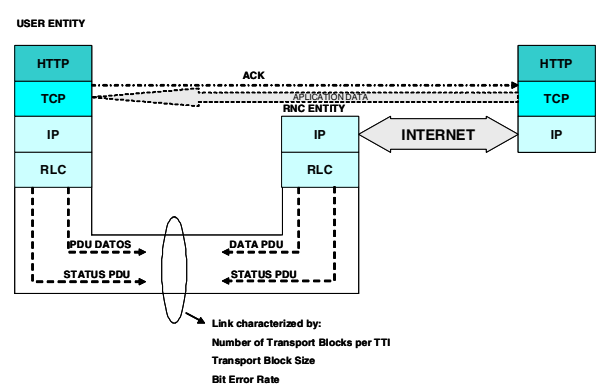

Fig. 1. RLC Evaluation Scenario and Simulation Model

In order to imitate WWW client traffic we have used an structural traffic model [16], where the activity of a WWW user session is reproduced by means of a hierarchical multilayered structure, whose parameters are statistically characterized to capture the most relevant real traffic features. In the simulations included in the present work, we have considered a permanent web session during which a client browses through different pages. Each pages consists of a bunch of objects which are downloaded by means of one or several TCP connections. Also, we have assumed that the web browser uses no more than two simultaneous connections, which matches the default behavior of most commercial web browser applications (e.g. Internet Explorer, Firefox, etc.). Once the page is downloaded, a random period of time is waited before triggering the download of the next page, to resemble the time devoted by the user to read the page. The parameters in our model (summarized in table II) have been extracted from traces captured both in academic and corporate networks [16] and are similar to those included in other characterizations [17].

\begin{tabular}{|l|l|l|l|}
\hline Parameter & Distribution & $\mu$ & $\sigma$ \\
\hline Page reading time (OFF) & Gamma & $46.8 s$ & $168.6 s$ \\
\hline Number of connections per page & Lognormal & 5.3 & 12 \\
\hline Simultaneous connections & Deterministic & 2 & - \\
\hline Connection size & Pareto & $5.6 \mathrm{kB}$ & $\begin{array}{c}\infty \\
(\alpha=1.77)\end{array}$ \\
\hline URI size & Lognormal & $\mu=364 \mathrm{~B}$ & $101 \mathrm{~B}$ \\
\hline
\end{tabular}

TABLE II

WEB CLIENT TRAFFIC MODEL PARAMETERS

The behaviour of the TCP connections generated according to the traffic model to download the page objects are simulated by using a detailed bidirectional model of TCP that includes all the main mechanisms of this protocol (connection setup and tear-down, slow-start, congestion avoidance, fast retransmit, fast recovery, Selective Acknowledgements, Time Stamps, etc.). The FACK [18] version of TCP, which uses selective acknowledgments to perform a more efficient recovery of lost packets, has been considered for the simulations because it is widely deployed at the present and is supported by a growing number of HTTP servers [19]. More details on the TCP configuration set are provided in the next section (See table III).

\section{Simulations AND Results}

Before presenting the simulation results it is necessary to define the metric used to evaluate the performance. In the related literature, different metrics such as TCP throughput and goodput, packet delay and RLC buffer size are considered. In the present study, given the selected traffic model, a metric providing information about the effective application-level data transmission rate is particularly interesting, because this reflects the user's perception of the system performance. Thus, results presented in this section are mainly based on the evaluation of the goodput. More specifically, we have used a weighed average of the page goodput defined by equation 1, where $S(p)$ and $T(p)$ are total data size and the download time of the $p-t h$ page, respectively.

$$
\widehat{A}_{\text {Gpage }}=\frac{\sum_{p=1}^{P} S(p)}{\sum_{p=1}^{P} T(p)}
$$

We have also considered other metrics such as the average page goodput (average of the goodputs of all the downloaded pages, considering the goodput of a page to be the ratio between its size and the time needed to download it), and the weighed and non-weighed averages of connection goodput. Results for these metrics are qualitatively similar and have not been included in this paper.

Using the simulation scenario described in section II-A with the default parameters summarized in table III, we have executed several tests to determine the impact of the RLC configuration in UMTS Internet access performance. In order to do determine the effect of a given parameter, different values has been simulated for it whereas the other parameters remain constant at their default values. For every analysed configuration, a total of 5 simulation runs have been executed using different random seeds. Each simulation run comprises the simulation of at least 5 millions of TCP connections generated according to the dynamics of the traffic model discussed in the previous section. The bandwidth of the radio access link, determined by the TTI $(20 \mathrm{~ms})$, the number of transport blocks per TTI (4), and the transport block size (80 bytes), is $128 \mathrm{kbps}$.

Figures 2, 3, 4 and 5 display the effect on the web traffic performance of the parameters related to poll triggering in the transmitter for different Bit Error Rates (BER). The order of magnitude of the $95 \%$ confidence intervals, which are not shown in the plots for the sake of clarity is about $10 \mathrm{~B} / \mathrm{s}$. Figs. 2, 3 and 4 show the effect of Timer Poll Periodic, Poll Window and Poll PDU, respectively, whereas fig. 5 shows the impact of different combinations of Timer Poll and Timer Poll Prohibit for TimerPollPeriodic $=0.96 s$, PollPDU $=192$ 


\begin{tabular}{|c|c|c|c|}
\hline \multirow{2}{*}{\multicolumn{2}{|c|}{$\begin{array}{c}\text { Radio Access Network Parameters } \\
\text { Physical and MAC layers }\end{array}$}} & \multicolumn{2}{|c|}{ TCP Parameters } \\
\hline & & MSS & 1460 Bytes \\
\hline Transport Block Size & 80 bytes & Max. Window Size & 16 kBytes \\
\hline Number of TB/TTI & 4 & Initial Window & 2 MSS \\
\hline TTI & $20 \mathrm{~ms}$ & Initial ssthreshold & $16 \mathrm{kBytes}$ \\
\hline \multicolumn{2}{|c|}{ RLC layer } & Delayed ACK timer & $0.2 \mathrm{~s}$ \\
\hline PDU Payload & 78 bytes & Max. Timeout & $64 \mathrm{~s}$ \\
\hline PDU Header & 78 bytes & Min. Timeout & $400 \mathrm{~ms}$ \\
\hline Window Size & 256 PDU & Default Timeout & $3 \mathrm{~s}$ \\
\hline SDU Buffer Size & unlimited & Timer granularity & $200 \mathrm{~ms}$ \\
\hline Timer Poll & 2 TTI & Maxburst & 4 MSS \\
\hline Timer Poll Periodic & 54 TTIs & Flavour & FACK \\
\hline Timer Poll Prohibit & 1TTI & \multicolumn{2}{|c|}{ Internet Parameters } \\
\hline Poll PDU & $217 \mathrm{PDU}$ & Latency & $50 \mathrm{~ms}$ \\
\hline Poll Window & $85 \%$ & & \\
\hline Timer Status Prohibit & Disabled & & \\
\hline Timer Status Periodic & Disabled & & \\
\hline Timer SDU Discard & Disabled & & \\
\hline Timer MRW Retransmit & 4 TTI & & \\
\hline
\end{tabular}

TABLE III

TCP/IP SIMULATION PARAMETERS

and PollWindow $=75 \%$. In all of them it can be observed that no outstanding differences exists between the different tested configurations, although those parameter combinations yielding a more frequent reception of status reports obtain better results. Naturally, this improvement is more pronounced when the Bit Error Rate is higher since the number of blocks to be recovered increases substantially.

When analysing the results, it should be considered that weighed average goodput may not approach the nominal link capacity (128kbps), mainly due to the fact that Web Traffic is a mixture of short and long lived TCP connections. Short lived TCP flows do not reach full download speed due to the slow-start algorithm of TCP congestion control, and therefore achieve much lower goodput than long-lived ones. To establish a reference, we have simulated the same traffic over an ideal $128 \mathrm{kbps}$ link, and the weighed average goodput obtained was 9.9 kbytes/s. Under this consideration, it is necessary to note that all the configurations analysed previously achieve a reasonable performance. In this sense, it must be observed that in any test the Poll Window parameter exceeds the $85 \%$ of the Window Size, which avoid RLC deadlocks and stall, thus preventing severe performance degradations.

The study of the receiver status reports triggering parameters Timer Status Periodic and Timer Status Prohibit has not been included in the present paper, but, due to their analogy to Timer Poll Periodic and Timer Poll Prohibit a similar result may be expected. Figure 6 shows the results corresponding to the evaluation of two parameters related to receiver-driven status reports transmission: Timer Status Prohibit and Out of order PDU threshold, henceforth PDU Threshold. These results has been obtained with all the transmitter side polltriggering mechanisms inactive except for Poll Window, which has been set to $95 \%$ to avoid deadlocks. Similarly to the transmitter-driven scheme case, better results are obtained for those parameter combinations causing a more frequent status reports generation, and again, the effect is stronger for a higher BER.

Finally, the influence of the SDU discarding timer has been also studied, and the results are presented in fig. 7 .

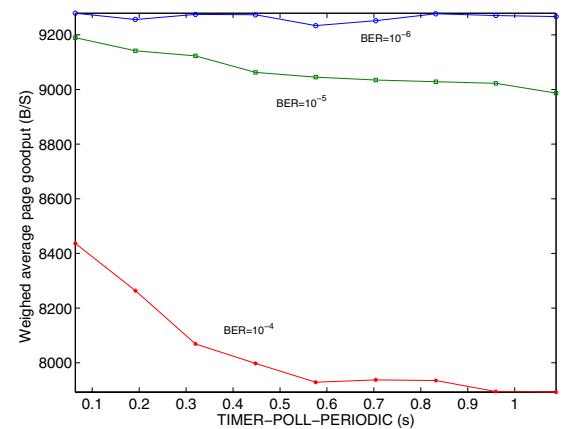

Fig. 2. Effect of Timer Poll Periodic in Web Browsing performance

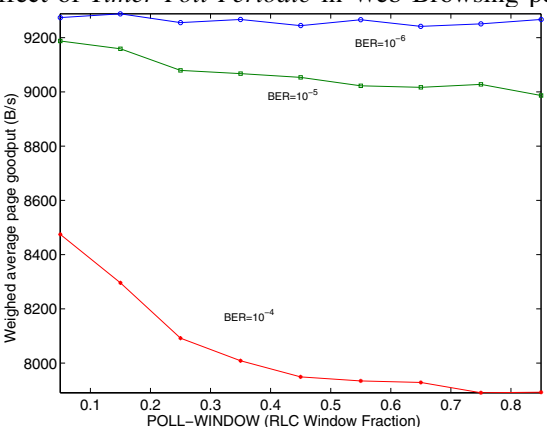

Fig. 3. Effect of Poll Window in Web Browsing performance

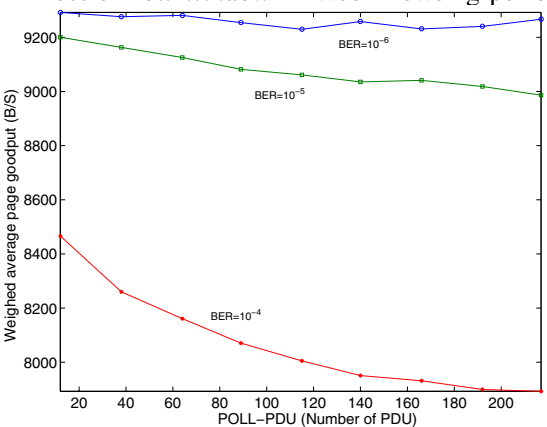

Fig. 4. Effect of Poll PDU in Web Browsing performance
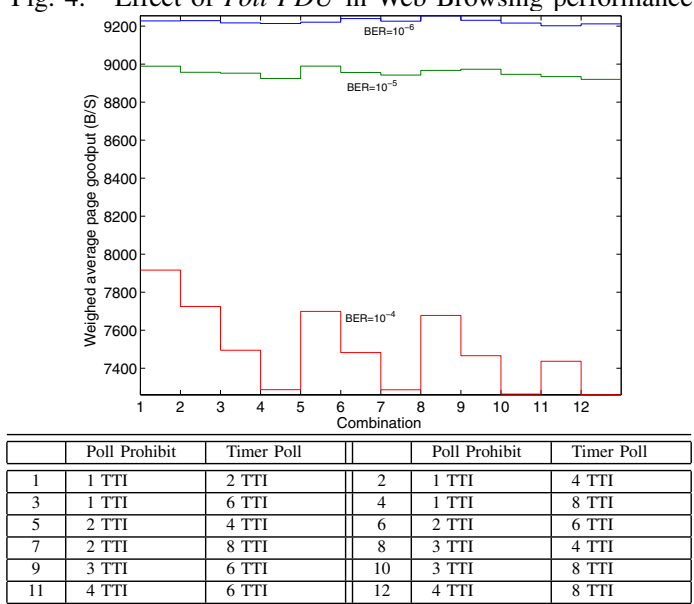

Fig. 5. Effect of Timer Poll and Timer Poll Prohibit in Web Browsing performance

In these simulations a transmitter-driven scheme has been used with Timer Poll Periodic=0.96s, Poll PDU=192 and Poll Window $=75 \%$. The SDU discarding mechanism elicits the elimination of those SDUs lingering in the RLC buffer without being successfully transmitted. This could improve 

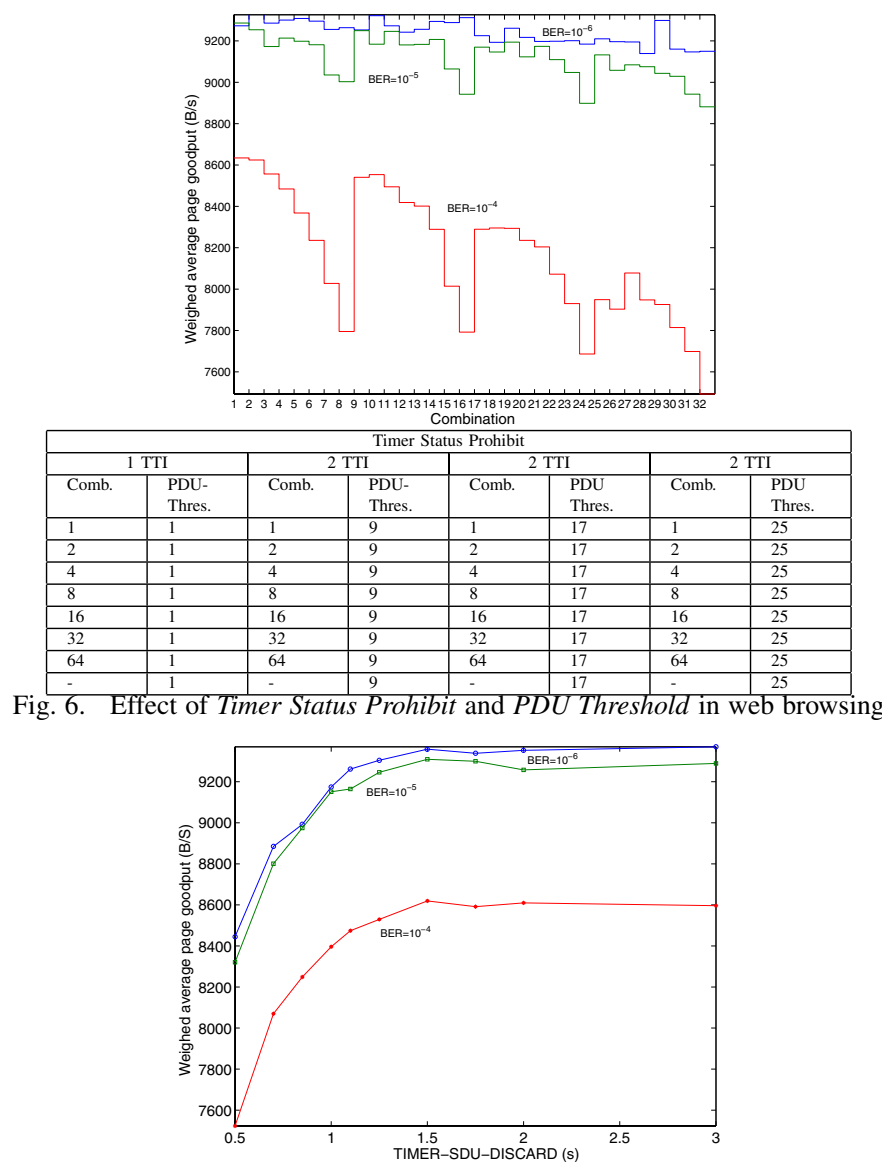

Fig. 7. Effect of Timer SDU Discard in web browsing

TCP performance under the consideration that after a certain amount of time these SDUs may have been retransmitted at transport level by TCP. This mechanisms reduces the delay suffered by the SDUs in the RLC buffer, limiting it, but, on the other hand, it provokes packet losses, which may reduce TCP performance. As can be observed in fig. 7 the SDU discard mechanisms does not introduce any improvement. Thus, for the considered traffic, the negative impact of packet loss in the link level is not at all compensated by the packet latency reduction. This is consistent with observations made in previous research found in the literature, cited in section II.

\section{CONClusions}

In this paper, the impact of the radio interface RLC layer configuration on the performance of the UMTS Internet access has been analysed. The study has mainly focused on the performance of web browsing because it is likely to continue being one of the leading services of the mobile Internet access. From our study it can be derived that RLC performance is acceptable for a relatively wide range of parameters, although for the evaluated traffic, which is rather asymmetric, those configurations bringing about a more frequent generation of status reports provide better results. Also, it has been proven that the activation of SDU discarding mechanisms to limit the amount of time that packets can await in the RLC buffer reduces the performance of the considered service.
The study included in this paper can be extended in several ways. Particularly, the effect of additional parameters and their interaction should be accomplished to complete the study. The evaluation of the effect of buffer size limits and its interaction with other parameters such as the SDU discarding timer is an interesting issue that will be explored in future research. It is also necessary to complete the study presented in this paper using more complex and realistic radio error models and test the performance of the different parameters configuration under a bursty packet error pattern.

\section{ACKNOWLEDGEMENTS}

This paper has been supported by Spanish public research fund program under project $\mathrm{N}^{\circ}$ TEL2003-07953-C02-01.

\section{REFERENCES}

[1] R. Chakravorty, S. Katti, J. Crowcroft, and I. Pratt, "Flow aggregation for enhanced TCP over wide-area wireless," in IEEE INFOCOM, May 2003.

[2] M. Methfessel, K. F. Dombrowski, P. Langendörfer, H. Frankenfeld, I. Babanskaja, I. Matthaei, and R. Kraemer, "Vertical optimization of data transmssion for mobile wireless terminals," IEEE wireless Communications, pp. 36-43, December 2002.

[3] P. Benko, G. Malicsko, and A. Veres, "A large-scale, pasive analysis of end to end TCP performance over GPRS," in IEEE INFOCOM, May 2004.

[4] R. G. Mukhtar, S. V. Hanly, and L. Andrew, "Efficient Internet traffic delivery over wireless networks," IEEE Communications Magazine, pp. 46-53, December 2003.

[5] M. Chan and R. Ramjee, "Improving TCP/IP performance over third generation wireless networks," in IEEE INFOCOM, May 2004.

[6] R. Ludwig and A. Gurtov, "Evaluating the eifel algorithm for TCP in a GPRS network," in European WirelessNFOCOM, February 2002.

[7] C. Casetti, M. Gerla, S. Mascolo, M. Sanadidi, and R. Wang, "TCP westwood: end to end bandwidth estimation for enhanced transport over wireless links," Wireless Networks, vol. 8, pp. 467-479, 2002.

[8] S. Heier, C. Ellerbrock, and M. Malkowski, "UMTS medium access control quality of service scheduling," in IEEEPIMRC 2002 - Personal Indoor and Mobile Radio Conference, vol. 5, September 2002.

[9] A. Baiocchi and F. Vacirca, "End to end evaluation of WWW and file transfer performance for UMTS-TDD," in IEEEGlobecom, 2002, pp. 737-741.

[10] R. Bestak, P. Godlewski, and P. Martins, "RLC buffer occupancy when using a TCP connection over UMTS," in PIMRC 2002 - Personal Indoor and Mobile Radio Conference, vol. 5, September 2002, pp. 2102-2106.

[11] F. Vacirca, A. D. Vendictis, A. Todini, and A. Baiocchi, "On the effect of ARQ mechanisms on TCP performance in wireless enviroments," in IEEEGlobecom, December 2003.

[12] C. Chiasserini and M. Meo, "Impact of ARQ protocols on QoS in 3GPP systems," IEEE Transactions on Vehicular Technology, vol. 52, no. 1, pp. 205-215, January 2003.

[13] "RLC protocol specification," 3GPP, Technical Specification 25.322v4.5.0 Release 4, June 2002.

[14] F. Lefevre and G. Vivier, "Optimizing UMTS link layer parameters for a TCP connection," in VTC Spring 2001, May 2001, pp. 2318-2322.

[15] C. Chiasserini and M. Meo, "A reconfigurable protocol setting to improve TCP over wireless," IEEE Transactions on Vehicular Technology, vol. 51, no. 6, pp. 1608-1620, November 2002.

[16] A. Reyes, E. González, E. Casilari, J. Casasola, and A. Diaz-Estrella, "A page-oriented WWW trafic model for wireless systems simulations," in Proceedings of 16th ITC, July 1999, pp. 1271-1280.

[17] H. Choi and J. Limb, "A behavioral model of Web traffic," in Proceedings of 7th IEEE International Conference on Networ Protocol (ICNP'99), september, 1999.

[18] M. Mathis and J. Mahdavi, "Forward acknowledgement: Refining TCP congestion control," in Proceedings of the ACM SIGCOMM'96, vol. 26, no. 4, 1996, pp. 281-291.

[19] A. Medina, M. Allman, and S. Floyd, "Measuring the evolution of transport protocols in the Internet," ACM Computer communication Review, vol. 35, no. 2, April 2005. 